\title{
Pulse Laser Observation of Electrostatically Extracted Ink Jet and its Application to Tone Reproduction
}

\author{
Member Kazuhisa Matsuo (Fukuoka Institute of Technology) \\ Member Takehiko Tomikawa (Kanagawa Institute of Technology) \\ Member Mamoru Nakajima (Fukuoka Institute of Technology)
}

\begin{abstract}
This paper describes an observation with use of pulse laser of behavior in detail of electrostatically extracted ink jet flight. In this trial, we have learned that randomly or asynchronously flying corpuscles can be photographed simultaneously by pulse laser in a bright field scattering image. An application of is also described with respect to continuous tone reproduction. Both cylindrical thread and flying corpuscles of ink jet are utilized in combination through a single outlet nozzle. The former is useful for higher density plottings and the latter for lower density plottings. Such a phenomenon of having plural states in the liquid stream is peculiar to the electrostatically extracted ink jet using dielectric ink.
\end{abstract}

Key words : Electrostatically extracted ink jet, Dielectric ink, Pulse laser, Tone reproduction

\section{Introduction}

Conventional methods of observation of ink jet flight have made use of high speed cameras and stroboscopes. These methods however require periodicity in ink jet flight. We have tried out a method of observation of the flying state of a continuous flow of liquid thread and ink corpuscles without periodicity beyond the flow in electrostatically extracted ink jet flight.

With regard to ink jet recording, various studies $^{(1) \sim(3)}$ have investigated in methods of tone reproduction of natural picture images. These methods, however, are based on the state of the periodically generated ink corpuscles. On the other hand an electrostatically extracted ink jet grows in plural states of its properties such as ink thread and ink corpuscles or mist. The former is useful for higher density plottings and the latter for lower density plottings that depend on the gray levels of reproduced images. Such a phenomenon of the distinct states in the liquid stream is peculiar to the electrostatically extracted ink jet using dielectric ink. We have studied the behavior of the electros. tatically extracted ink jet and its application to the smooth tone reproduction of natural picture images ${ }^{(4)}$. This paper describes the tone reproduction by utilizing both ink thread and corpuscles of ink jet in combination through a single outlet nozzle.

With the foregoing in mind, detailed behavior of ink jet flight was observed with pulse laser beam. An observation of flying ink jet is also described with respect to the laser beam photography. Photograph the liquid stream, which is generated randomly or asynchronously over the transition state of flow, has been difficult in the past. In this trial, however, we have learned that the randomly flying objects can easily be photographed simultaneously by the aid of laser irradiation.

This paper describes a new method with the use of pulse laser beam of observation of phenomena in electrostatically extracted ink jet flight. Furthmore, a case where these phenomena are effective for tone 
reproduction is presented.

\section{Transition state of ink jet}

Flying ink, in this trial, is possible to transit its state from the liquid thread into the atomizing corpuscles and then into the scattering mist depending on the applied voltage, and on the properties of ink as well. The volume and the length of the liquid thread can be made variable by controlling the applied voltage. These variations of ink jet are quite useful for applying to tone reproduction of images. Next to the liquid thread, the ink jet is gradually atomized into corpuscles, and then dispersed due to repulsive force among electrified corpuscles of ink. In the scattering region at the end of the ink jet, ink corpuscles are considered to be randomly dispersed by the turbulence of factors such as electrostatic field or air resistance. Thus the states of ink jet thought to be of practical interest must be the liquid thread region and the atomizing region since recorded density is dark in the liquid thread and light in the atomizing corpuscles.

However it was considered necessary that ink adhering to the recording paper after being atomized into corpuscles must be recorded within a given area.

The liquid thread length can be controlled by the applied voltage (voltage of nozzle to electrode) or ink pressure as shown in Figs $4 \sim 6$.

\section{Laser observation system of ink jet}

Microscopic photographing of flying ink can normally be done with a stroboscope or a high-speed camera, but not for the randomly or asynchronously flying corpuscles. The stream of electrostatically extracted ink jet has a continuous flow in the liquid thread region but not in the region where ink transforms into corpuscles in flight. Our approach to photographing such irregularly flying corpuscles is by means of directing a laser beam to this transition stage. Fig. 1 shows our experimental photographing system of ink jet. The nozzle is fixed directly onto the inkpot and is applied voltage $V_{b}$.

The nozzle is made of a stainless pipe, $680 \mu \mathrm{m}$ in outer diameter, $380 \mu \mathrm{m}$ in inner diameter, and 15 $\mathrm{mm}$ in length. The tip of the nozzle is cut at $30^{\circ}$ to

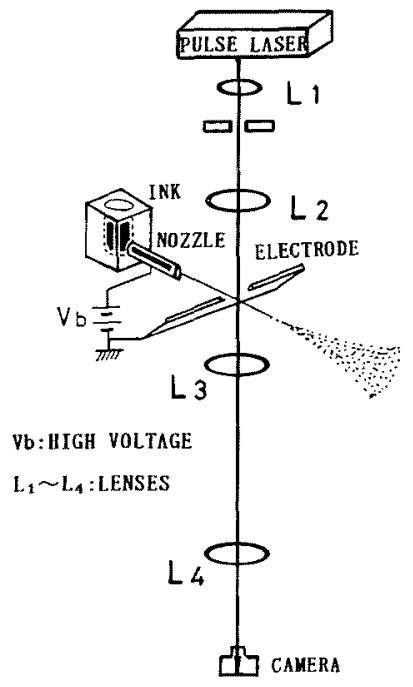

Fig. 1. Observation system of ink jet by pulse laser.

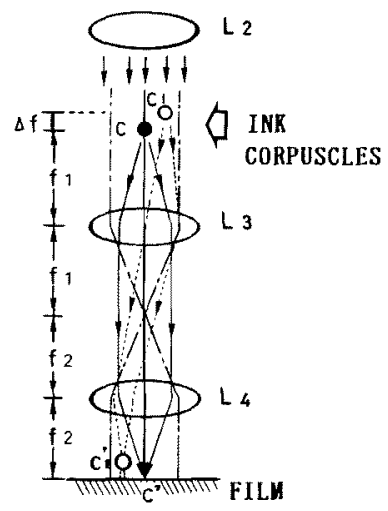

Fig 2. Details of observation on ink corpuscles by pulse laser.

the center line to improve the response of ink jet. Two electrodes of $0.8 \mathrm{~mm}$ in diameter, $3 \mathrm{~mm}$ apart, are positioned perpendicularly $2 \mathrm{~mm}$ from the nozzle. Ink files by applying voltage between nozzle and the electrodes. Thus ink flies through the center of a $3 \mathrm{~mm}$ distance between electrodes.

For the light source, pulse laser is used. A laser beam is directed through the optical system $L_{1}-L_{4}$, and ink flight must be photographed on the film. The ink jet generator should be positioned between $L_{2}$ and $L_{3}$ because laser beam is a plane wave at 


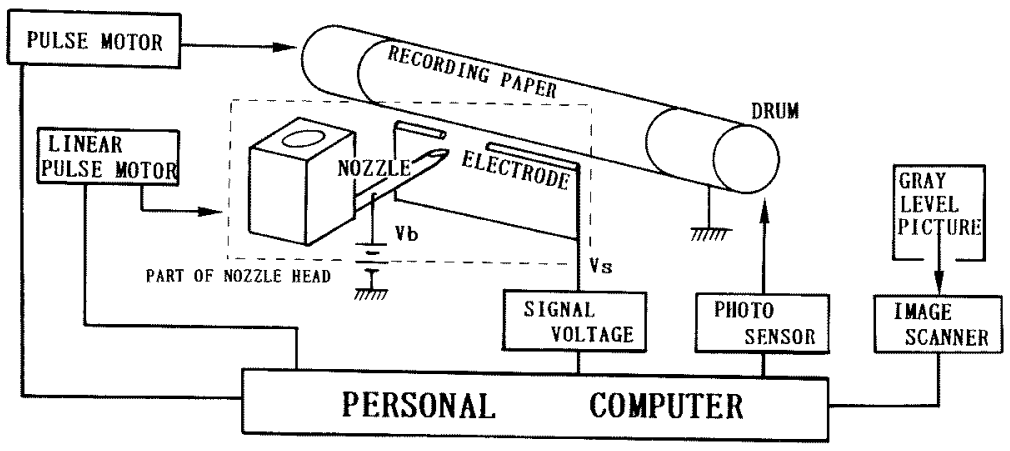

Vb:DC BIAS Vs:SIGNAL VOLTAGE OF PICTURE

Fig. 3. Experimental system of tone reproduction.

lenses $L_{2}$ and $L_{3}$. Fig. 2 shows the details of our optical system. The ink jet generator of nozzle should be positioned within a minute distance $\Delta f$ from the focal point $f_{1}$ on the object side of the lens $L_{3}$. The ink corpuscles on the object side of the lens $L_{3}$ are projected through lenses $L_{3}$ and $L_{4}$ by plane waves, and the plane waves and scattered light from corpuscles form an interfering image $C_{1}$ on the image side, and the image is recorded in the form of hologram in a larger size than the actual size. The corpuscle $C$ at the focal point is recorded on the film surface $C^{\prime}$ as a minute shadow image in the actual size. On irradiating the pulse laser $(0.4 \mu$ s width), an inline hologram can be photographed on the film as a bright field scattering image due to incident light and scattering light diffracted by ink corpuscles through $L_{1}$ to $L_{4}$.

In such a case, corpuscles on the object side are recorded as high density image but as they deviate more from the focal point, the image becomes larger and low in density.

\section{Tone reproduction of experimental system}

Fig. 3 shows a block diagram of our experimental system. The electrode system at the nozzle head portion is shown in Fig. 1. To the nozzle is applied DC bias $V_{b}$. The electrodes are positioned $2 \mathrm{~mm}$ from the nozzle tip. To these electrodes is applied picture signal voltage $V_{s}$ which is related to the gray levels of reproduced images. A grounded drum $70 \mathrm{~mm}$ in diameter is positioned $1 \mathrm{~mm}$ away from the electrodes resulting in a differential voltage of ( $V_{b}$ $-V_{s}$ ) between the nozzle and electrodes. The ink flight varies in accordance with the Coulomb-force caused by this differential voltage. A recording is thus on the recording paper around the drum.

The applied voltage as recording condition is $V_{b}$ $=2.0 \mathrm{kV}, V_{s}=500 \mathrm{~V}$ and ink pressure is $H=3 \mathrm{~mm}$.

\section{Observation of ink jet by pulse laser beam}

Figs $4 \sim 6$ shows an experimental photograph by our method using pulse laser beam.

Fig. 4 shows the effect of applied voltage with the static ink pressure level $H=8 \mathrm{~mm}$, and the electrode positioned $2 \mathrm{~mm}$ from the nozzle tip and recording paper $1 \mathrm{~mm}$ from the electrode. Applied voltages are from $2.8 \mathrm{kV}$ to $3.7 \mathrm{kV}$. Ink scatters less as the applied voltage is increased. On the recording paper, when the applied voltage is low, scattering is wider in atomized state, whereas when the applied voltage is high, the scope of scattering is small. As the applied voltage is increased, liquid thread also becomes longer. When the applied voltage is further increased, several ink jets are produced.

Fig. 5 shows the case where the distance between the nozzle and electrode is changed to $3 \mathrm{~mm}$. The state of ink flight is about the same as in the case of the distance of $2 \mathrm{~mm}$ However the point where the ink transforms into corpuscles is farther away from the tip and this point is presumed to be beyond the electrodes.

Tone reproduction was considered possible by 


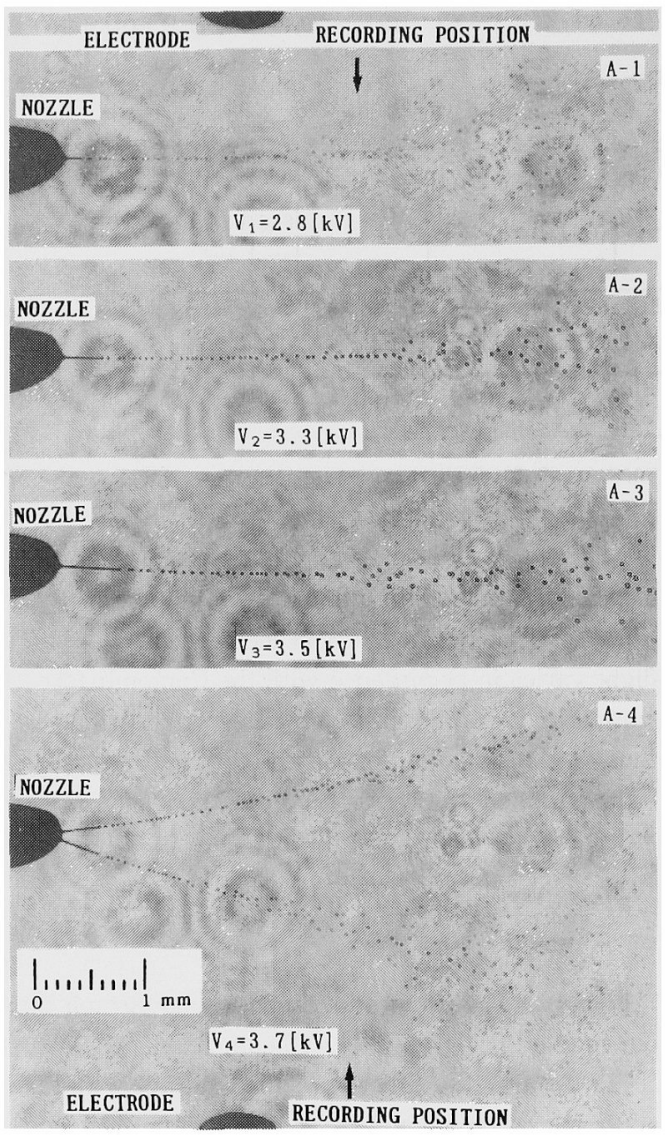

Distance of nozzle to electrode is $2 \mathrm{~mm}$, ink pressure $(H)$ is $8 \mathrm{~mm}$.

Fig. 4. Observation of ink jet by pulse laser.

using the liquid thread region by increasing applied voltage for the high density area and by using the ink corpuscles region by decreasing applied voltage for the low density area. The applied voltage however must be within the voltage range which produces stabilized ink jet flight but does not lead to flight of multiple ink jets. Also, the position at which ink jet flight scatters occurs past the electrodes even when the nozzle to electrode distance is changed.

When reproduction of an image is to be made, the recording paper is positioned $1 \mathrm{~mm}$ from the electrodes. This is an experimental position but this position is the most suitable in recording images.

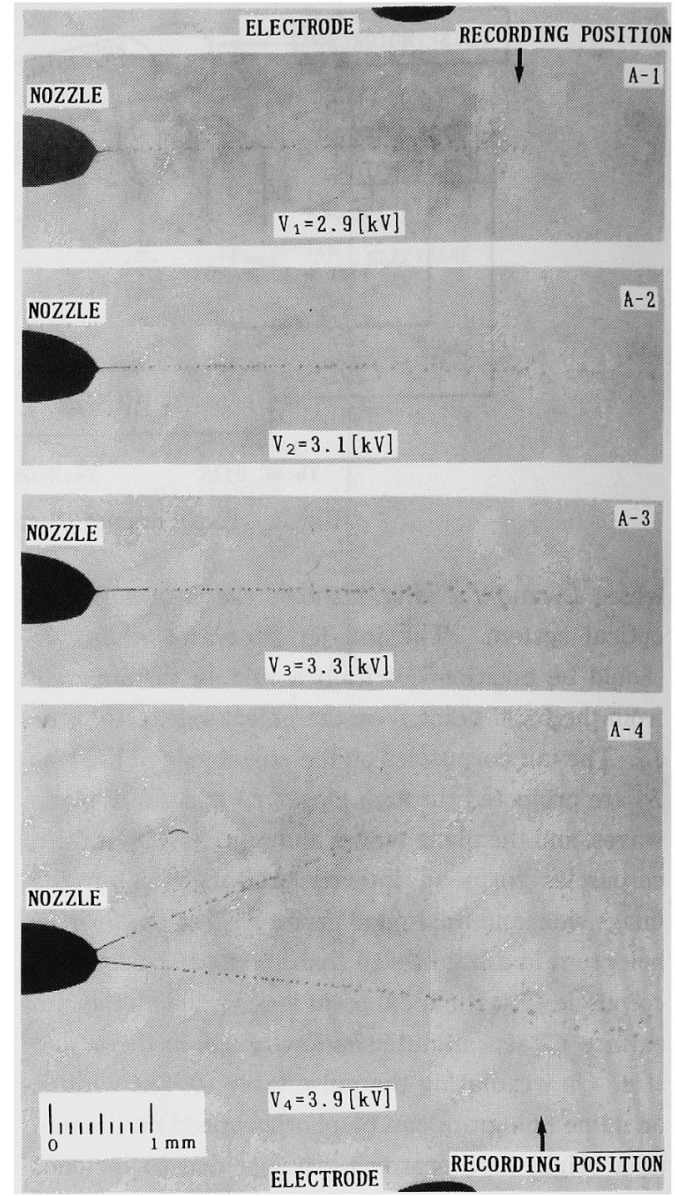

Distance of nozzle to electrode is $3 \mathrm{~mm}$, ink pressure $(H)$ is $8 \mathrm{~mm}$.

Fig. 5. Observation of ink jet by pulse laser.

Fig. 6 shows the effect of ink pressure when the applied voltage is $3 \mathrm{kV}$ and the distance between the nozzle and electrodes is $3 \mathrm{~mm}$. Ink pressure is from $3 \mathrm{~mm}$ to $15 \mathrm{~mm}$. Higher the ink pressure is, the greater the meniscus becomes. Liquid threads also become longer. The length of liquid thread can be made variable by changing ink pressure. The recording paper is positioned in relation to the electrodes, and as in Figs 4 and 5, difference in density is clearly shown.

It was presumed possible to control the state of ink adhering on the recording paper by changes in 

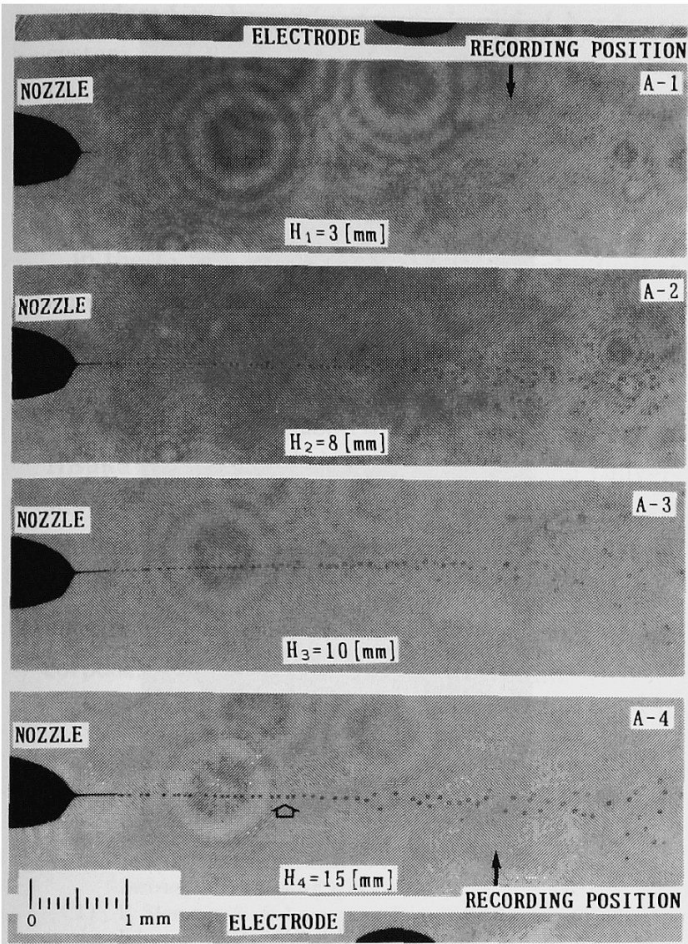

Distance of nozzle to electrode is $3 \mathrm{~mm}$, applied voltage $(V)$ is $3 \mathrm{kV}$.

Fig. 6. Observation of ink jet by pulse laser.

liquid thread length due to changes in ink pressure. In tone reproduction, it was presumed possible to reproduce high density area by increasing ink pressure, and to reproduce low density area by decreasing ink pressure.

Fig. 7 shows an enlargement of the portion indicated by arrow in the inline hologram of Fig. 6 $(A-4)$. Fig. 8 shows that the diameter of a corpuscle can be determined by analyzing the inline hologram of a liquid droplet. The average spherical size is approximately $11 \sim 17 \mu \mathrm{m}$, when ink pressures are 3 $\sim 15 \mathrm{~mm}$, followed by the linuma's formula ${ }^{(5)}$, which is quite similar to that obtained by Hertz $z^{(3)}$. As to diameters of ink jet, higher the ink pressure is, the larger ink corpuscles are; but the effect of the applied voltage is small. For example, in the case of applied voltage of $V=3.0 \mathrm{kV}$, and ink pressure of $H=10 \mathrm{~mm}$, the diameter obtained is about $16 \mu \mathrm{m}$.

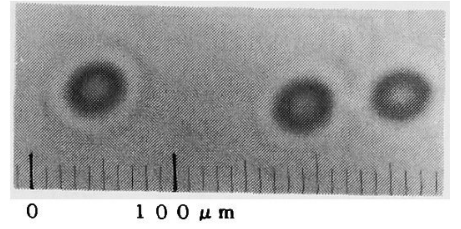

Fig. 7. Portion indicated by arrow in the inline hologram in Fig. 6(A-4).

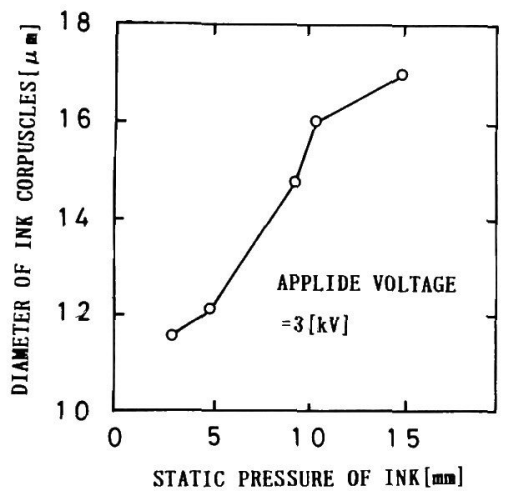

Fig. 8. Characteristics of corpuscles diameter vs. static ink pressure.

In high quality reproduction of images, it is thought advantageous for the spherical size of the ink corpuscle to be minute. Consequently, it is thought to be better to make static ink pressure low. With our method, although the nozzle inner diameter is large at $380 \mu \mathrm{m}$, a minute ink corpuscle diameter of $11 \mu \mathrm{m}$ can be obtained with static ink pressure of $H=3 \mathrm{~mm}$ and nozzle clogging can be prevented. With other methods, for the nozzle inner diameters of $30 \sim 50 \mu \mathrm{m}$, the corpuscle diameters are $100 \sim 150$ $\mu \mathrm{m}$, resulting in ink corpuscles about two to three times larger than the nozzle diameters.

Fig. 9 shows the effect of ink pressure on liquid thread length. The nozzle-to-electrode distance is $3 \mathrm{~mm}$ in ( $\mathrm{a}$ ) and $2 \mathrm{~mm}$ in ( $\mathrm{b}$ ). Ink pressure is $H=$ $5,8,15 \mathrm{~mm}$ for both ( $\mathrm{a}$ ) and ( b ). Higher the ink pressure is, the longer the liquid thread length is in both cases. The starting voltage for ink flight and the applied voltage for several ink jets to fly are shifted by about $100 \mathrm{~V}$.

It is seen from these results that the liquid thread is lengthened as the applied voltage is increased.

電学論 C, 113 巻 5 号, 平成 5 年 


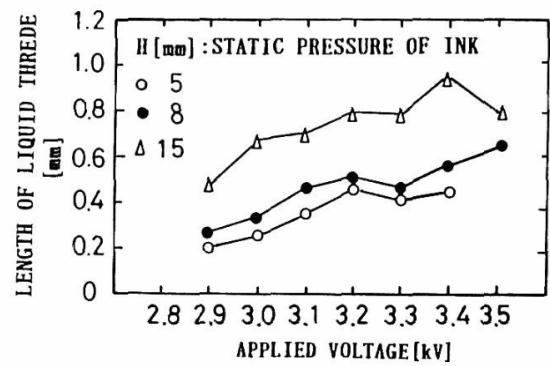

(a) Distance of nozzle to electrode is $3 \mathrm{~mm}$.

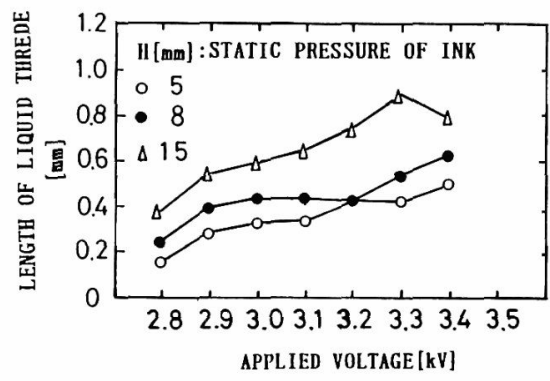

(b) Distance of nozzle to electrode is $2 \mathrm{~mm}$.

Fig. 9. Characteristics of liquid thread length vs. applied voltage.

In practice, the recording paper is set $1 \mathrm{~mm}$ from the electrodes. Consequently, when the applied voltage is increased, the liquid thread becomes longer and the distance to the recording paper becomes shorter and tone reproduction of the high density area is made convenient. When the applied voltage is decreased, the liquid thread becomes shorter and effective reproduction of the low density area.

\section{Conclusion}

In this paper, details of behavior of ink jet flight have been made clear by a new observation method using dielectric ink of electrostatically extracted ink jet. Application of the phenomena to tone reproduction was experimented and its validity was proved. Observation by pulse laser beam of ink jet flight made possible the photographing on one frame a continuous flow of liquid thread with periodicity and atomized ink corpuscles without periodicity beyond the flow. As a result, changes in liquid thread length, dispersion position of ink corpuscles,

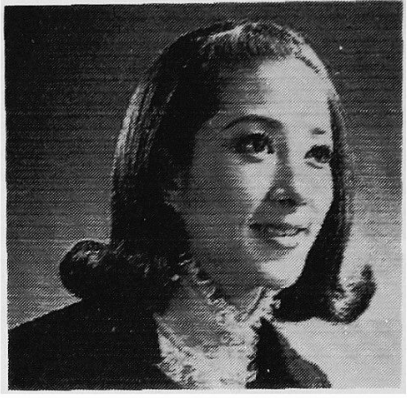

(a) Girl

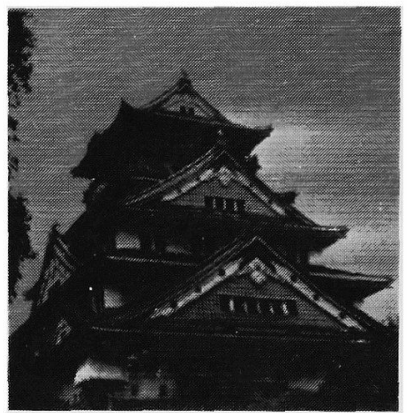

(b) Castle

Fig. 10. Print examples.

and spherical size of ink corpuscles due to changes in applied voltage and static ink pressure have been made clear. On the basis of the foregoing results, an application to tone reproduction was tried.

Tone reproduction of image was considered possible by varying the state of ink adhering to the recording paper placed at a fixed position, which was brought about by varying the length of liquid thread by controlling the applied voltage or static ink pressure.

For this paper, we used the method in which applied voltage, easier to control, was varied. In tone reproduction of image, using lower static ink pressure was more advantageous for reproduction of the lower density area.

An experimental example of the image reproduction is shown in Figs 10 ( a ), a girl, and ( b ), a castle.

In these figures, the input images of $(a)$ and $(b)$ are a photographic print and an artificial painting, respectively. As a result, the tone image reproduced by utilizing the transition state of ink jet is 
considered to be useful for a simplified hardware system. Meanwhile, an observation scheme of flying ink jet is introduced with respect to the laser beam photography. We have learned that the irregularly flying objects can be photographed by pulse laser as a bright field scattering image.

In the future, the authors intend to make further improvements on the experimental systems and to make measurements of flying ink jet at various velocities.

We are deeply indebted to Professor Kazuyosi Hirakawa, Faculty of Engineering, Kyushu University, for his invaluable advice and to Professor Akitoshi Murakami, Faculty of Engineering, Kyushu University, for his kind guidance in photographing the ink flight by laser and measurement of ink corpuscles diameter from inline hologram.

(Manuscript received June 24, '92)

\section{References}

(1) C. H. Hertz \& A. Mansson: "Electric Control of Fluids Jets and its Application to recording Devices", Rev. Sci. Instruments, 43, No. 3, 413 (1972)

(2) E.Stemme \& S. Larsson: "The Piezoelectric Capillary Injector", IEEE Trans. Electron Device, ED-20, No. 1, 14 (1973)

( 3 ) C. H. Hertz \& B. Samuelsson: "Ink Jet Printing of Photographic Quality Color Images”, J. Imaging Tech., 15, 141 (1989)

(4) K. Matsuo, T. Tomikawa, T. Katayama \& M. Nakajima: "A Study of Tone Reproduction and Laser Observation of Electrostatically Extracted Ink Jet", Proc. of 7th IS \& T, Vol. 2, p. 377 (1991)

(5) T. Iinuma, et al.: Laser Diagnostics and Modeling of Combustion, p. 71 (1987) Springer-Verlag

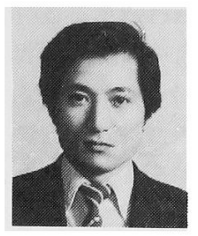

\section{Kazuhisa Matsuo (Member)}

He is a lecture in the Department of Communication \& Computer, Fukuoka Institute of Technology. He received the B.S. degree from Fukuoka Institute of Technology in 1971. He has been interested in application of ink jet.

$\mathrm{He}$ is a member of IEEE.

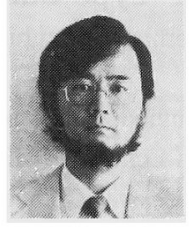

Takehiko Tomikawa (Member)

$\mathrm{He}$ is a associate professor in the Department of Electrical Engineering, Kanagawa Institute of Technology. He received the $\mathrm{ME}$, and $\mathrm{Ph}$. D. degrees from Shizuoka University in 1979 and 1982, respectively. His present research activeties involve image processing and its applications.

$\mathrm{He}$ is a member of IEEE.

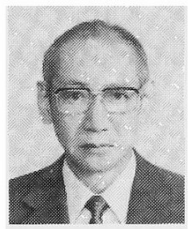

\section{Mamoru Nakajima (Member)}

He is a professor in the Department of Communication \& Computer, Fukuoka Institute of Technology. $\mathrm{He}$ received the $B . S$. degree from Tohoku University in 1949. He has been interested in application of ink jet. 